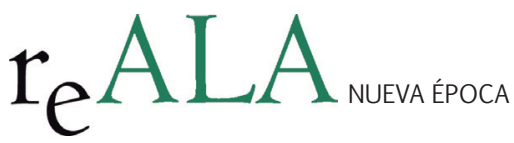

REALA, n 2, julio-diciembre 2014, ISSN: 1989-8975

DOI: http://dx.doi.org/10.24965/reala.voi2.10192

\title{
El pretendido blindaje autonómico de competencias municipales tras la reforma de la Administración Local
}

\author{
Pilar Navarro Rodríguez \\ Profesora de Derecho Administrativo. Universidad Loyola Andalucía \\ pnavarro@uloyola.es \\ Manuel Zafra Víctor \\ Profesor Titular de Ciencia Política y Administración Pública. Universidad de Granada
} manuelzafravictor@gmail.com

Recibido: 25 de mayo de 2014 Aceptado: 20 de junio de 2014

\section{Resumen}

Ante la gran incertidumbre provocada por la LRSAL, hasta la fecha cuatro CCAA han aprobado diferentes normas (Galicia una Ley, Castilla y León y Andalucía sendos Decretos-Leyes, y País Vasco una Circular) de "blindaje" de competencias municipales, con el fin de precisar y aclarar algunos de los temas para los que la Ley no ofrece una interpretación clara o una aplicación inequívoca. El denominador común de esta normativa autonómica es tratar de desactivar el propósito de la LRSAL: reducir la calificación de competencias municipales impropias a las que no cuenten con habilitación del Legislador Sectorial (autonómico) anterior o posterior a la promulgación de la Ley, y condicionar en materia de Servicios Sociales, Sanidad y Educación la calificación de esas competencias a la modificación de la financiación autonómica. En este artículo se abordan estas cuestiones, partiendo del análisis del Anteproyecto de Ley, y pasando por las importantes consideraciones sobre el mismo realizadas por el Dictamen del Consejo de Estado.

Palabras clave

Administración Local, Autonomía Local, Competencias Municipales, Competencias Autonómicas.

\section{The Intended Shield for Regional Municipal Powers after Reform of Local Government}

\begin{abstract}
Given the large uncertainty caused by the LRSAL, to date, four have adopted different standards CCAA (a law Galicia, Castile and León and Andalusia two separate decree-laws, and the Basque Country Circular) "shield "of municipal powers, to refine and clarify some of the issues for which the Act does not provide a clear unambiguous interpretation or application. The common denominator of this regional legislation is to try to disable the purpose of LRSAL reduce the improper classification of municipal competencies that do not have enabling Sector Legislator ( regional) before or after the enactment of the Act, and condition in field of Social Services, Health and Education qualification of those powers to the modification of the regional financing. In this article these issues, based on the analysis of the Draft Law, and going through the important considerations about the same made by the State Council Opinion addressed.
\end{abstract}

Keywords

Local Administration, Local Self-Government, Municipal Competencies, Autonomic Competencies 
1. Sometimiento de la autonomía local a las exigencias de estabilidad presupuestaria. El intento de supresión virtual de la autonomía local mediante la legislación básica./ 2. La transformación del Legislador Básico en Legislador Armonizador con la misma pretensión de supresión virtual de la Autonomía Local./ 3. Interpretación del artículo 25.2 en relación con el 7.4: el régimen jurídico de las competencias distintas de las propias o atribuidas por delegación. La regulación en el Anteproyecto de Ley./ 4. El contenido armonizador de la Ley. Las bases estatales no ocupan el espacio constitucionalmente reservado al legislador autonómico pero le imponen, de la pluralidad de ejercicios de sus competencias, uno determinado./ 5. Una premisa fundamental para la cabal comprensión de la racionalización y sostenibilidad local: el Estado financia pero las Comunidades Autónomas fijan el alcance de las competencias municipales./ 6. Competencias impropias, competencias complementarias y cláusula general de competencia./ 7. Medios para ajustar el desajuste entre ingresos y gastos de los entes locales./ 8. La interpretación del régimen jurídico de las competencias distintas de las propias en la Ley. Retroactividad o irretroactividad en la LRSAL./ 9. Normativa autonómica de blindaje de competencias municipales frente a la LRSAL./ 10. Bibliografía.

\section{SOMETIMIENTO DE LA AUTONOMÍA LOCAL A LAS EXIGENCIAS DE ESTABILIDAD PRESUPUESTARIA. EL INTENTO DE SUPRESIÓN VIRTUAL DE LA AUTONOMÍA LOCAL MEDIANTE LA LEGISLACIÓN BÁSICA.}

Tal y como señalamos en su momento', frente a lo que sucedió con la última gran reforma de la Ley 7/1985, de 2 de abril, Reguladora de las Bases de Régimen Local' (en adelante, también, la “LRBRL"), la que vino como consecuencia de la promulgación de la Ley 57/2003, de 16 de diciembre, de Medidas para la Modernización del Gobierno Local' ${ }^{3}$, el origen de la reforma que aprueba la Ley 27/2013, de 27 de diciembre, de Racionalización y Sostenibilidad de la Administración Local ${ }^{4}$ (en adelante, también, la "LRSAL”), no es tanto la evaluación negativa del funcionamiento de la Administración local como la necesidad de poner en marcha el programa de reformas de la Administración española que viene impuesto por compromisos políticos y obligaciones jurídicas contraídas con Europa5, constitucionalmente integradas en nuestro ordenamiento, al menos en lo referido a la necesidad de contener el gasto público y el déficit y de mejorar la eficiencia administrativa, en virtud de la reforma del artículo 135 de la Constitución Española (en adelante, también, la “CE”) aprobada mediante la Ley Orgánica 2/2012, de 27 de abril, de Estabilidad Presupuestaria y Sostenibilidad Financiera'.

Este es el motivo por el cual el tratamiento que la autonomía local7 (singularmente la municipal) recibe en la LRSAL, es el de un sector o materia sometida a las exigencias de estabilidad presupuestaria como consecuencia de la crisis económica ${ }^{8}$.

De esta forma, el Estado ha pretendido, desde la legislación básica (con la aprobación de la LRSAL), controlar

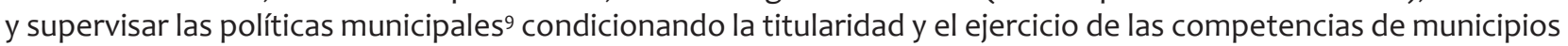
y provincias.

Y lo ha hecho, en primer lugar, reduciendo la protección constitucional proporcionada por la garantía institucional, mediante tres vías, a saber:

1. La rebaja de las materias de obligatoria referencia para los legisladores sectoriales a la hora de atribuir competencias a los municipios.

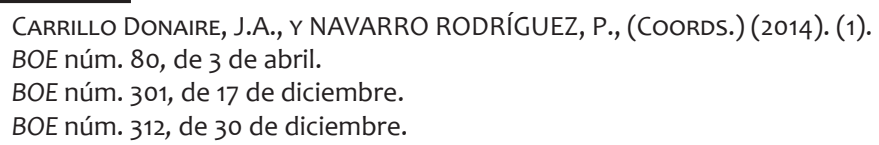

8 En este contexto se enmarcan los trabajos impulsados por el Instituto Nacional de Administración Pública (INAP) y por la Comisión para la Reforma Administrativa que están en la base de la reforma local y que se mueven en la línea reduccionista de la racionalización que inspira el principio de "una Administración, una competencia", que mayestáticamente proclama la Exposición de Motivos de la Ley.

9 Y ello porque, a nuestro entender, y pese a que el carácter bifronte de la autonomía local y el ejercicio de competencias legislativas sobre el régimen local concierne tanto al Estado como a las Comunidades Autónomas, es mucho más sencillo para el Ejecutivo central comenzar el programa de reformas por el escalón inferior, la Administración local, que auto-recetarse una cura de adelgazamiento a sí mismo o enfrentarse a las enormes dificultades jurídicas y políticas que conllevaría cualquier movimiento de reordenación orgánica y de recentralización funcional de las Comunidades Autónomas. 
2. La supresión de competencias complementarias, y

3. La reducción de la cláusula general de competencia prevista en el artículo 25.1 de la LRSAL a una mera declaración de capacidad jurídica para obrar.

Ahora bien, como quiera que la disminución de la garantía institucional operada por el Estado central como Legislador Básico no le aseguraba que las Comunidades Autónomas no pudiesen atribuir competencias a los municipios, el Legislador Estatal ha cruzado con la LRSAL la línea del Legislador Básico para situarse como Legislador de Armonización, tal y como se desarrolla en el siguiente apartado.

\section{LA TRANSFORMACIÓN DEL LEGISLADOR BÁSICO EN LEGISLADOR ARMONIZADOR CON LA MISMA PRETENSIÓN DE SUPRESIÓN VIRTUAL DE LA AUTONOMÍA LOCAL}

Tal y como acabamos de señalar, con el objetivo principal de tratar de evitar que las comunidades autónomas puedan atribuir competencias a los municipios, con la LRSAL el Estado cruza la línea del Legislador Básico para situarse como Legislador de Armonización: de esta forma, al tiempo que priva a los municipios de determinadas competencias en servicios sociales, sanidad y educación, impone a las comunidades autónomas la titularidad de las competencias municipales ejercidas hasta ahora en estas materias.

Y no acaban aquí las pretensiones armonizadoras del Legislador Estatal, sino que obliga también al Legislador Sectorial (Autonómico) a la elaboración de una memoria que constate el menor gasto generado por una asignación diferenciada de competencias en comparación con su ejercicio centralizado desde el nivel autonómico. De forma que si esta segunda posibilidad arrojara menor coste, la respectiva consejería de la comunidad autónoma en cuestión tendría prohibida la atribución de competencia.

Pero es más, tampoco estaría a disposición del correspondiente Legislador Sectorial la delimitación en negativo de las competencias municipales, ya que debe habilitar a los municipios en positivo, precisando funciones y especificando materias, es decir de los posibles ejercicios autonómicos de una competencia de su exclusiva titularidad, el Estado le impone uno determinado.

Por estos motivos, entendemos que se puede afirmar de manera clara y meridiana que cualquier regulación autonómica que propicie la libertad municipal para modular el ejercicio de sus funciones y potestades y determinar así el alcance de sus competencias, encuentra en la LRSAL prohibiciones claras o requisitos onerosos.

Partiendo de esa base, y a la luz de las cuestiones antes descritas, cabe inferir que el propósito de la LRSAL pasa por la eliminación virtual de la autonomía municipal, asunto que en un primer momento se intentó en los diversos anteproyectos y proyecto de Ley con la radicalidad de la introducción de un coste estándar para la prestación de servicios, y ante las objeciones del Consejo de Estado ${ }^{10}$, más indirectamente, mediante el coste efectivo como guía en el ejercicio tanto de las competencias municipales como de las competencias provinciales.

El resultado es evidente: sacrificio de la democracia en aras de una presunta eficiencia sobreponiendo unas entidades locales de legitimación indirecta como la provincia a las de directa legitimación como los municipios ${ }^{11} \mathrm{o}$ sustrayendo al control de la oposición y fiscalización de los grupos minoritarios decisiones tan importantes como la elaboración de planes económico-financieros.

Ante las limitaciones que el bloque de constitucionalidad impone al Estado para conseguir la unilateral racionalización y sostenibilidad de la autonomía local, el Legislador Estatal no sólo rebaja el mínimo protegido constitucionalmente ${ }^{12}$, sino que pretende vetar o dificultar que lo eleven las comunidades autónomas.

\section{INTERPRETACIÓN DEL ARTÍCULO 25.2 EN RELACIÓN CON EL 7.4: EL RÉGIMEN JURÍDICO DE LAS COMPETENCIAS DISTINTAS DE LAS PROPIAS O ATRIBUIDAS POR DELEGACIÓN. LA REGULACIÓN EN EL ANTEPROYECTO DE LEY ${ }^{13}$}

Quizás la cuestión más controvertida de la LRSAL, sea la interpretación del artículo 25.2 en relación al artículo 7.4, es decir, la distinción entre competencias propias y las competencias distintas de las propias.

La polémica surge con (por) las opuestas lecturas sobre la naturaleza jurídica del listado contenido en el apartado 2 del artículo 25. Si se atiende a una primera interpretación literal, la expresión "en todo caso" alude a

\footnotetext{
10 En el Dictamen número 567/2013, de 26 de junio, del Consejo de Estado. Sobre este tema, vid., in extenso, ZAFRA VÍcTOR, M., (2013b). (16). Sobre este tema, vid., in extenso, ZAFRA VícTOR, M., (2014). (17). Vid., ZAFRA VÍCTOR, M., (2013a). (15).
} 
un mínimo que obliga al Legislador Sectorial a la atribución de competencias a los municipios; si la interpretación atendiera, por ejemplo, al Dictamen del Consejo de Estado, la enumeración de competencias constituiría un máximo vinculante para los Legisladores Sectoriales que sólo podrían modular el alcance de las materias allí fijadas pero no atribuir competencias propias en otras materias (distintas).

El término máximo significativamente no aparece en el razonamiento del Consejo que expresa la nueva regulación en los siguientes términos:

(...) el anteproyecto introduce una importante novedad a través de la modificación del artículo 25 de la LRBRL, que pasa de establecer un listado abierto de competencias susceptibles de ser legalmente reconocidas a los Municipios y a enunciar tales competencias de forma exhaustiva y aparentemente cerrada. Ello determina que no quepa en el sistema que el anteproyecto configura la atribución como competencias propias de otras distintas de las incluidas en dicha relación, lo que constituye una significativa diferencia con respecto al actual modelo de asignación de competencias, en el que cualquier ley sectorial puede reconocer al Municipio como propias competencias para actuar en cualquier ámbito.

El cambio en el régimen jurídico de las competencias propias sitúa en calidad de impropias todas las competencias municipales atribuidas por los Legisladores Sectoriales (no se olvide, principalmente autonómicos) en materias no contenidas en el artículo 25.2 y solo podrán los municipios continuar en su ejercicio si cumplen con los requisitos exigidos por el artículo 7.4. Ante la posible inconstitucionalidad esgrimida por las comunidades autónomas, el Consejo de Estado defiende su conformidad con la Constitución Española en los siguientes términos:

(...) no cabe objetar que el Estado lleve a cabo una reducción del ámbito competencial de los Municipios, pues, siempre que respete el núcleo básico e intangible que define la autonomía local como tal, puede en el ejercicio de dicha competencia ampliar o estrechar la esfera de actuación de las Corporaciones Locales. Y si como consecuencia de tal operación se produce una alteración del marco competencial de los Municipios tal y como ha sido definido por las Comunidades Autónomas, habrán de ser estas las que acomoden su legislación a lo dispuesto con carácter básico por el legislador estatal. Puede este, en definitiva, efectuar una redefinición del ámbito competencial municipal, por más que ello obligue al legislador autonómico a introducir en su ordenamiento los ajustes necesarios.

En efecto, el Consejo de Estado recurre a una paráfrasis para eludir que el Estado fija un máximo, y en su lugar señala el carácter exhaustivo y cerrado del listado sosteniendo la constitucionalidad de una reducción de las competencias municipales pues salvaguarda el núcleo básico e intangible (por cierto, tampoco emplea el término mínimo).

A nuestro parecer, con esta interpretación jurídica el Consejo de Estado (que ha sido ratificada en un nuevo y reciente Dictamen de 26 de mayo de $2014^{14}$ con ocasión del conflicto en defensa de la autonomía local planteado por el Alcalde de Barcelona, donde el Consejo se reafirma en su criterio sobre el máximo) incurre en un doble error:

- El primero y más elemental: el Estado no atribuye competencias a los municipios, fija las materias donde, según la distribución constitucional de competencias, Estado y Comunidades Autónomas, las asignarán a los municipios. Recurriendo a una distinción elaborada por el Tribunal Constitucional, si el legislador de régimen local atribuyera competencias a los municipios en materias de titularidad autonómica, trascendería su carácter de Estado en sentido estricto para convertirse en Estado en sentido amplio, estaría, pues, ocupando el espacio constitucionalmente reservado a las Comunidades Autónomas. Sin embargo el Dictamen continuamente alude a la prerrogativa del Estado (en sentido estricto) para reducir el ámbito competencial de los municipios (folio 61) o la reducción del ámbito de las competencias propias del Municipio (folio 26), en clara contradicción con la naturaleza de las bases que limitan su radio de acción a la enumeración de materias donde estado y comunidades autónomas deberán asignar competencias a los municipios.

- $\quad$ Este primer error lleva al segundo: como el Estado no reconoce competencias a los municipios sino que fija el mínimo de una enumeración de materias, las comunidades autónomas disponen de libertad para, una vez respetado ese mínimo, ampliar el elenco competencial de los municipios.

Pese a la renuencia del Consejo de Estado a reconocer el cambio radical de su interpretación, la nueva regulación impone un máximo que las comunidades autónomas no pueden elevar. La posibilidad de ejercer

14 Dictamen núm. 338/2014, de 26 de mayo, del Consejo de Estado. 
competencias impropias tiene prevista una vía distinta: el municipio debe solicitarlas a condición de no incurrir en duplicidad y garantizar la sostenibilidad financiera; la comunidad autónoma, mediante informe deberá acreditar la inexistencia de duplicidad y el interventor la garantía de sostenibilidad. Por tanto, no hay, en sentido estricto, atribución autonómica de competencia. El propio Consejo lo dice a la luz de la redacción del artículo 7.4 del Anteproyecto de Ley:

(... ) tras la entrada en vigor de la ley proyectada tales competencias ejercidas hasta ahora como propias pasarán a quedar fuera de ese ámbito del artículo 25 de la LRBRL, convirtiéndose así en lo que el anteproyecto denomina "competencias impropias". Por tanto, esas competencias, referidas a materias respecto de las cuales las Comunidades Autónomas ostentan competencias ya previstas en las correspondientes leyes autonómicas, solo podrán continuar siendo ejercidas por los correspondientes Municipios si, efectuada la evaluación prevista en la disposición transitoria novena, se constata que cumplen con los requisitos que impone el nuevo artículo 7.4 de la LRBRL.

En efecto, la disposición transitoria novena del Anteproyecto acotaba con precisión el concepto de competencia impropia de la siguiente forma:

Las competencias distintas de las previstas en los artículos 25.2 y 27 de la Ley $7 / 85$, de 2 de abril reguladora de las Bases de Régimen Local que hasta la entrada en vigor de esta Ley vinieran ejerciendo los Municipios sólo podrán seguir ejerciéndolas si cumplen con los previsto en el apartado 4 del artículo 7 de la Ley $7 / 85$.

La conclusión, por tanto, aparece inequívoca a nuestro parecer: fuera del listado del artículo 25.2 las competencias serán o delegadas o impropias; el legislador autonómico no podrá atribuir competencias propias en otras materias de las allí enumeradas.

Conviene distinguir, no obstante, la valoración positiva que el Consejo hace sobre este tema de las severas objeciones formuladas al coste estándar, para comprender correctamente el juicio que el anteproyecto merece al órgano consultivo. La disposición transitoria novena sujetaba el ejercicio de competencias impropias a una evaluación conforme al coste estándar; si el resultado pusiera de manifiesto el incumplimiento de los requisitos contemplados en el artículo 7.4, mediante informe del interventor municipal, el municipio dejará de prestarlas. A su vez, el cumplimiento de estos requisitos se encomendaba a informe de la comunidad autónoma acreditando la inexistencia de duplicidades y al del interventor en lo relativo a sostenibilidad. El Consejo de Estado no cuestiona la existencia de ambos informes, tampoco la finalidad, sus reparos se dirigen a la indeterminación y vaguedad jurídica del concepto duplicidad y a la inadecuación del informe del interventor, al tiempo que recomienda la conveniencia de concebir el informe no sobre competencias puntuales sino sobre el conjunto de la hacienda municipal, de ahí la propuesta para que sea la comunidad autónoma la encargada de emitirlo.

Como consecuencia de todo ello, el Gobierno central acoge todas las indicaciones del Consejo; suprime el coste estándar, asigna el informe sobre duplicidades a la administración competente por razón de la materia y el de sostenibilidad a la titular de la tutela financiera. Ahora bien, como decíamos más arriba, no cuestiona el fondo del asunto: ni la noción de competencia impropia (el cambio es meramente nominal, competencias distintas de las propias) ni los necesarios y, ahora, vinculantes informes autonómicos. Por tanto, el listado del artículo 25.2 constituye un máximo y las materias no contempladas que fueran de competencia autonómica, solo podrán serlo de competencia municipal a condición de cumplir los requerimientos de estabilidad y sostenibilidad.

Por tanto, el cambio más sustantivo provocado por el Dictamen del Consejo de Estado es la supresión del coste estándar; las competencias impropias, pese a su la nueva paráfrasis, continúan teniendo relevancia jurídica y el concepto de ejecución simultáneo permite su mejor distinción con respecto al de complementariedad. No se trata de subestimar los efectos pero las imposiciones establecidas por el legislador estatal sobre los autonómicos quedan justificadas: fuera de la enumeración del artículo 25.2, el legislador sectorial no podrá atribuir competencias a los municipios. Teniendo en cuenta que el listado presenta más cercanía a una enumeración de competencias que a uno de materias, el margen de maniobra para el correspondiente legislador autonómico disminuye, una disminución que se acentúa al exigir la determinación de la competencia municipal para evitar la ejecución simultánea del mismo servicio por otra administración tal como se prevé en el apartado 5 del artículo 25. 


\section{EL CONTENIDO ARMONIZADOR DE LA LEY. LAS BASES ESTATALES NO OCUPAN EL ESPACIO CONSTITUCIONALMENTE RESERVADO AL LEGISLADOR AUTONÓMICO PERO LE IMPONEN, ENTRE LA PLURALIDAD DE EJERCICIOS DE SUS COMPETENCIAS, UNO DETERMINADO}

A juicio del Consejo de Estado, la reforma de las bases limita su recorrido a rebajar el mínimo garantizado por la Constitución a la autonomía municipal, una decisión sin tacha de constitucionalidad porque, pese a la reducción, el municipio resulta reconocible como institución. Sin embargo, el reproche de constitucionalidad no se limita al menoscabo operado sobre la autonomía municipal, la repercusión más negativa la experimenta el legislador autonómico e, indirectamente, el propio municipio. En la medida que el listado del artículo 25.2 constituye una enumeración exhaustiva y cerrada, un auténtico techo (máximo) en lugar de un suelo (mínimo), el legislador estatal define la competencia autonómica en positivo, imponiéndole uno de los posibles ejercicios: en un importante elenco de materias (servicios sociales, educación y sanidad) no podrá atribuir competencias propias a los municipios y en las fijadas en el artículo 25.2 deberá hacerlo conforme a criterios extraordinariamente restrictivos.

El resultado final es evidente: el legislador básico reduce la autonomía municipal (en principio ninguna tacha de constitucionalidad cabe oponer a ello) pero impide a los legisladores sectoriales autonómicos que eleven el mínimo, en primer lugar circunscribiendo las competencias propias al listado del apartado 2 del artículo 25 y, en segundo lugar, obligando a la elaboración de una memoria económica sobre el impacto económico de la atribución y a la precisión en la función y la especificidad en la materia a la hora de atribuir las competencias a los municipios, una operación, como se señalaba antes, casi imposible a la vista del detalle del listado. Hay un dato relevante a tener en cuenta que culmina las condiciones exigidas por el legislador estatal: obligando a la delimitación precisa con el fin de evitar duplicidad o ejecución simultánea del mismo servicio por otra administración, el legislador autonómico pierde la opción para delimitar en negativo la competencia municipal, deberá hacerlo en positivo hasta el punto que la pormenorización de la competencia municipal acabe privando de autonomía al municipio en la medida que se verá obligado a aplicar o ejecutar las leyes autonómicas, en lugar de a desarrollarlas en una relación normativa afín a la mantenida entre bases estatales y legislación autonómica de desarrollo. Este sería, a nuestro juicio, el riesgo alojado en el apartado 5 del artículo 25.

Desde nuestro punto de vista, el procedimiento adoptado por el legislador estatal excede su condición de legislador básico, ya que con el título competencial de las bases del régimen jurídico de las administraciones públicas no tiene habilitación constitucional para delimitar en positivo las competencias autonómicas; quizás pudiera calificarse como exceso de las bases el detalle del listado del artículo 25.2, pues más que materias y funciones genéricas, ofrece precisión en ambos ámbitos; pero parece claro que no hay desmesura de las bases al constreñir el ejercicio de las competencias autonómicas a uno de los múltiples ejercicios a su disposición; en este caso se evidencian las pretensiones armonizadoras de la ley, el legislador estatal no ocupa el espacio constitucionalmente reservado al autonómico sino que, invocando presuntas razones de interés general, garantía de eficiencia para evitar duplicidades y aseguramiento de la sostenibilidad, le obliga a un determinado ejercicio de una competencia que no resulta usurpada pero sí desnaturalizada.

\section{UNA PREMISA FUNDAMENTAL PARA LA CABAL COMPRENSIÓN DE LA RACIONALIZACIÓN Y SOSTENIBILIDAD LOCAL: EL ESTADO FINANCIA PERO LAS COMUNIDADES AUTÓNOMAS FIJAN EL ALCANCE DE LAS COMPETENCIAS MUNICIPALES}

La causa primera de estas pretensiones armonizadoras quizás haya de buscarse en un desajuste estructural de la inserción de los entes locales en el estado autonómico. A pesar del artículo 142 de la Constitución Española el Tribunal Constitucional ha erigido en garante de la suficiencia financiera al Estado. Y ante esta situación, las comunidades autónomas se han apresurado a proclamar que no tienen la obligación de financiar a municipios y provincias y, sutilmente, han recurrido al término "colaboración", normalmente articulada mediante recursos afectados en forma de subvenciones (ver estatutos reformados, catalán y andaluz). La patología institucional de hacer al Estado el financiador surge del desfase entre financiación local y competencias locales porque el nivel competencial de los municipios depende, en última instancia, de las comunidades autónomas. La finalidad fundamental de los principios de racionalización y sostenibilidad es acompasar recursos económicos y ejercicio de competencias para deshacer la anomalía denunciada por el municipalismo: competencias impropias y gastos de suplencia. La reforma local adopta un tono armonizador para imprimir coherencia a competencias y financiación. Este era el sentido de fijar por decreto el coste estándar para la prestación de servicios. Descartado este medio tras los reparos del Consejo de Estado, permanece, no obstante, el propósito de jerarquizar el ejercicio de las competencias municipales dando prioridad a las competencias propias y a los servicios obligatorios, asegurando que las competencias delegadas y los convenios sólo serán efectivos si prevén los recursos correspondientes y, finalmente, sometiendo las competencias distintas de las propias y las atribuidas por delegación, a unos requisitos severamente restrictivos.

REALA, n’ 2 julio-diciembre 2014, ISSN: 1989-8975 - DOI: http://dx.doi.org/10.24965/reala.v0i2.10192 
El profesor Velasco Caballero no aprecia inconstitucionalidad en un orden de prelación para el ejercicio de las competencias municipales ${ }^{15}$, ya que a su juicio estaría amparado por el título competencial que permite al estado, según la jurisprudencia del Tribunal Constitucional (STC 214/89) establecer el régimen jurídico del sistema competencial.

La duda que entendemos que surge de esta posición no es tanto si la elasticidad infinita de la garantía institucional autoriza este orden de prelación, la afectación más directa la soporta el legislador autonómico obligado al reconocimiento de competencias municipales siguiendo un restrictivo procedimiento impuesto por el estado para el ejercicio de una competencia de su exclusiva titularidad. Que el Legislador Sectorial deba delimitar en positivo mediante habilitación la competencia municipal y tenga prohibida su delimitación en negativo, como hemos tenido ocasión de exponer antes, se halla más cerca de la armonización, teniendo en cuenta los fines de estabilidad y sostenibilidad que se quieren alcanzar, que de concretar en esta materia el régimen jurídico de las administraciones públicas.

Es más, abonaría nuestra opinión, como veremos más adelante, el hecho de que el artificio está resultando fallido con la normativa autonómica de desarrollo, toda vez que el Legislador Estatal no dispone de las competencias autonómicas, ni las relativas a régimen local ni las sectoriales de atribución de competencias a los municipios.

\section{COMPETENCIAS IMPROPIAS, COMPETENCIAS COMPLEMENTARIAS Y CLÁUSULA GENERAL DE COMPETENCIA}

Con elogiable rigor analítico, el profesor Velasco señala que la inadecuada distinción entre competencias propias o impropias (o distintas de las propias) en la LRSAL, encubre otra igualmente injustificable: entre competencias específicas y competencias genéricas, entre las que, a su vez, la LRSAL establece la jerarquía en su ejercicio dando prioridad a las primeras sobre las segundas.

Y como ha apuntado el profesor Díaz Lema "cualquier indeterminación competencial constituye un elemento extraño en la perspectiva contable del texto" "16. Este fue el motivo, al parecer, para la supresión del artículo 28 que legitimaba al municipio para el ejercicio de competencias complementarias y la modificación a la baja de la cláusula general de competencias del artículo 25.1. Díaz Lema resalta la dimensión contable del entonces anteproyecto, perfectamente apreciable en la Ley finalmente aprobada, a la búsqueda del ajuste estricto entre ingresos y gastos.

Dicho de otra forma, irónicamente, las bases estatales, aparentemente, satisfacen las quejas municipalistas de competencias impropias y gastos de suplencia, ya que a partir de la entrada en vigor de la LRSAL, contarán con financiación para el ejercicio de sus competencias, claro está a costa de limitar radicalmente las competencias municipales, hasta el punto que el apartado 1 del artículo 25 deja de dar cobertura al municipio descubridor de competencias y limita su alcance jurídico a una declaración de capacidad jurídica circunscribiendo sus efectos al listado del apartado 2 del mismo artículo. Este decisivo cambio veta la iniciativa municipal para el ejercicio de una competencia de no contar con habilitación legal o recibir doble informe favorable de la comunidad autónoma sobre inexistencia de ejecución simultánea y garantía de sostenibilidad.

Por tanto, la interpretación que la cláusula había recibido por parte del profesor Carro Fernández-Valmayor ${ }^{17}$ (y del Tribunal Supremo e incluso del Tribunal Constitucional) queda desactivada: en ausencia de ley sectorial no cabe competencia municipal en las materias del artículo 25.2, no hay, pues, vis expansiva de la cláusula general indistinguible ahora de la genérica capacidad de obrar recogida en el artículo 5 de la LRBRL y la expresión en el ámbito de sus competencias no resulta superflua ni contradictoria sino coherente con la dicción literal del nuevo precepto:

El Municipio, para la gestión de sus intereses y en el ámbito de sus competencias, puede promover actividades y prestar los servicios públicos que contribuyan a satisfacer las necesidades y aspiraciones de la comunidad vecinal en los términos previstos en este artículo.

De manera gráfica podría afirmarse a la luz del tenor literal de este precepto, que los municipios ven afectada sensiblemente la incondicionalidad de sus recursos ya que cada competencia cuenta con la financiación precisa para su ejercicio.

\section{MEDIOS PARA AJUSTAR EL DESAJUSTE ENTRE INGRESOS Y GASTOS DE LOS ENTES LOCALES}

Para alcanzar estos objetivos contables resumidos, por cierto, en el lema "una administración, una competencia", la LRSAL procede a definir las competencias municipales en términos análogos a los servicios

15 Vid., in extenso, VELASCO CABALLERO, F., (2014). (13).

16 DÍAZ LEMA, J.M., (2013). (3).

17 CARRO FERNÁNDEZ-VALMAYOR, J.L., (1999/2000). (2).

REALA, n 2 julio-diciembre 2014, ISSN: 1989-8975 - DOI: http://dx.doi.org/10.24965/reala.v0i2.10192 
obligatorios; teniendo en cuenta que el listado de materias del artículo 25.2 y el de servicios obligatorios del artículo 26.2 presenta sustanciales coincidencias, el Legislador Estatal intenta ajustar la financiación a las competencias reconocidas en la Ley y exige que cualquier ampliación o concreción competencial corra a cargo del Legislador Sectorial correspondiente.

La intención resulta tan determinada que el apartado 4 del artículo 25 ha recibido una taxativa interpretación, bien para considerarla adecuada, bien para mostrar su inadecuación. El precepto contempla la existencia de una memoria económica que refleje el impacto de la competencia atribuida sobre los recursos económicos de las administraciones públicas afectadas y obliga a la previsión de la dotación económica necesaria para asegurar la suficiencia de las entidades locales.

Y el párrafo final del referido precepto contiene los motivos de la polémica: "en ningún caso puede conllevar un mayor gasto de las Administraciones Públicas".

El profesor Díaz Lema lo interpreta como un principio que debe ponderar la viabilidad de otro, el de descentralización, de tal manera que si la asignación diferenciada de competencias a los municipios provocara un coste más elevado que su ejercicio centralizado (normalmente autonómico), el Legislador Sectorial tendría prohibido asignar la competencia. El principio de sostenibilidad financiera primaría sobre el de descentralización y proximidad.

En cambio, frente a esa interpretación, hay otras que defienden lo contrario, destacando entre todas ellas la del Gabinete Jurídico de los Servicios Centrales de la Junta de Andalucía, que considera inconstitucional ${ }^{18}$ subordinar el núcleo esencial, identificador del municipio, al mayor o menor gasto que implique el desarrollo de una determinada competencia.

Dicho de otra forma: condicionar la asignación de una competencia específica a su impacto económico puntual desvinculado del conjunto de las haciendas de las administraciones involucradas -municipal y autonómica, fundamentalmente- supone erigir injustificadamente el coste económico en criterio decisivo para el reparto competencial entre niveles de gobierno y, por tanto, el artículo 25.4 incurriría en inconstitucionalidad.

El Gabinete Jurídico de la Junta de Andalucía estima, sin embargo, que la inconstitucionalidad hallaría fundamento en la lesión provocada contra la autonomía local orillando, a nuestro juicio, el efecto más directo: la limitación del ejercicio de una competencia autonómica, o más claramente, el veto a un determinado ejercicio. Curiosamente el escrito de los Servicios Centrales del Gabinete Jurídico de la Junta de Andalucía continúa con la argumentación en contra del carácter armonizador de la Ley cuando, como hemos tratado de razonar con anterioridad, el primer afectado negativamente por el precepto es el legislador sectorial autonómico, el Legislador Sectorial estatal no se halla vinculado por la legislación básica, como así lo dejó establecido la STC 240/2006, donde se niega función constitucional a la LRBRL y se descarta su inclusión en el bloque de constitucionalidad.

Las aspiraciones armonizadoras de la Ley expresadas gráficamente en el lema, "una administración, una competencia", confieren plena coherencia a los apartados 2, 4 y 5 del artículo 25. El Estado define con detalle las competencias y dificulta al legislador autonómico su concreción o ampliación, bien mediante una memoria económica que demuestre coste menor para que la descentralización permita la sostenibilidad, bien exigiéndole precisión en la estructura de la competencia (precisión en las funciones y especificidad en la materia) para evitar la duplicidad.

\section{LA INTERPRETACIÓN DEL RÉGIMEN JURÍDICO DE LAS COMPETENCIAS DISTINTAS DE LAS PROPIAS EN LA LEY. RETROACTIVIDAD O IRRETROACTIVIDAD EN LA LRSAL}

Las objeciones planteadas por el Consejo de Estado obligaron al Gobierno a modificaciones importantes en el Anteproyecto de Ley pero, una vez introducidos dichos cambios, no queda despejada una duda fundamental: ¿Pueden las comunidades autónomas una vez suprimida la disposición transitoria novena atribuir competencias a los municipios en materias distintas a las enumeradas en el artículo 25.2? Tal como en esta disposición se preveía e interpretó en su dictamen el Consejo de Estado, la promulgación de la Ley ¿convierte las competencias propias hasta entonces ejercidas por los municipios en impropias si salen fuera del listado contenido en el artículo 25.2? Los interrogantes plantean un tema fundamental: la retroactividad o irretroactividad de la Ley.

Antes de abordar este decisivo aspecto, conviene reparar en la posición de la Federación Española de Municipios y Provincias (en adelante, también, la “FEMP”) y su interpretación del artículo 25.2.

18 En el Recurso de Inconstitucionalidad interpuesto por la Junta de Andalucía frente a la LRSAL. 
La FEMP, en sus Alegaciones presentadas al Anteproyecto de Ley ${ }^{19}$, coincide con el Consejo de Estado en el carácter tasado de la enumeración (contenida en) del artículo 25.2. Pese a la dificultad para entender su argumentación desde un punto de vista jurídico, puede resultar políticamente comprensible; las comunidades autónomas se han mostrado extraordinariamente renuentes a reconocer competencias plenas y enteras a los municipios, un análisis de las competencias municipales atribuidas por las comunidades autónomas revela un evidente déficit de calidad cuando no caen en la habitual remisión diabólica a la LRBRL que, a su vez, remite al correspondiente legislador sectorial. De ahí que los municipios hayan buscado la protección del Estado frente a las Comunidades Autónomas, e incluso el Tribunal Constitucional justificó el contenido de las bases estatales para no dejar a la libre decisión de cada comunidad autónoma la determinación de las competencias municipales (STC 214/89).

Sin embargo la LRSAL da un giro a la premisa: el Estado rebaja el mínimo de la autonomía municipal e impide o dificulta a las comunidades autónomas que lo amplíen. La FEMP, no obstante, conserva el subconsciente político anterior a la reforma de la ley, y considera que una ampliación de competencias sin la financiación pertinente condena a los municipios a las competencias impropias y los gastos de suplencia. Durante la tramitación parlamentaria de la Ley, la Federación solicitó la supresión de una enmienda que proponía la inclusión de un apartado 6 en el artículo 25 para aclarar equívocos, una vez descartada la disposición transitoria novena del Anteproyecto, sobre el carácter cerrado (máximo) o abierto (mínimo) del listado recogido en el apartado 2 del artículo 25 . El precepto que se quería añadir como apartado seis en el texto aprobado por la Comisión el 7 de noviembre decía lo siguiente:

Cuando, por Ley, las Comunidades Autónomas en sus respectivos ámbitos competenciales atribuyan a los municipios competencias propias en materias distintas a las previstas en el apartado 2 del presente artículo, deberán tener en cuenta los criterios señalados en los apartados 3, 4 y 5 anteriores.

La FEMP pedía su supresión porque supondría un cambio radical en el sentido de la Ley, pues las comunidades autónomas legitimarían la práctica de las competencias impropias. La representación municipal y provincial parece entender que las competencias municipales propias se limitan a las enumeradas en el artículo 25.2, al afirmar en sus Alegaciones lo siguiente:

Este párrafo modifica el eje central de la Ley en cuanto al sistema de atribución de competencias, ya que deja la puerta abierta a que las CC.AA puedan atribuir como propias a las EELL distintas de las referidas en el Artículo 25.2 (...). La introducción de este apartado 6 supone dar carta de naturaleza, nuevamente en el Régimen Local, a las competencias impropias. Permitiría que los municipios tuvieran que atender competencias autonómicas que las Comunidades Autónomas les encomendaran, y además sin financiación para ello, sin un lazo mínimo y sin la garantía regulada en esta Ley.

Ha sido justamente el éxito de la propuesta de la FEMP y la desaparición del apartado 6 lo que ha llevado a algún autor, destacando el profesor Medina Guerrero ${ }^{20}$, a mantener el criterio interpretativo del listado como un máximo fuera del cual no cabe asignación autonómica de competencia a los municipios.

Sin embargo y pese a la inevitable polémica, otras interpretaciones han justificado la legitimación del Legislador Sectorial autonómico para atribuir competencias municipales. Así, el profesor Velasco Caballero la fundamenta en varias razones. La primera, una lectura conforme al bloque de constitucionalidad y a la jurisprudencia constitucional; la segunda por (ante) la imposibilidad de otorgar un significado distinto al mismo concepto de competencia propia; la Ley reconoce a Navarra competencia para asignar a los municipios competencias propias. La primera razón lleva al profesor Velasco a recordar la evidencia de un listado de materias, no de competencias y a señalar, oportunamente, que el legislador básico, al disminuir el listado, no reduce el elenco de competencias municipales. Cuando este es su propósito, como en el caso de los servicios sociales, aunque con técnica legislativa deficiente y virtualmente inconstitucional, lo prevé en una disposición transitoria.

Por su parte, el profesor Jiménez Asensio, emplea también una doble argumentación para sostener el carácter irretroactivo de la Ley ${ }^{21}$. En primer lugar, al igual que el profesor Velasco, el bloque de constitucionalidad impide al estado ocupar el espacio constitucionalmente reservado a las comunidades autónomas; en segundo lugar, si la entrada en vigor de la ley supusiera (implicara) que las competencias propias de los municipios redujeran su número a las acotadas en el artículo 25.2, nos encontraríamos, a su entender, no ante una reforma, por importante

19 Vid., FEDERACIÓN ESPAÑOLA DE MUNICIPIOS Y PROVINCIAS (2013). (4).

20 Vid., MEDINA GUERRERO, M., (2014). (8).

21 Vid., JIMÉNEZ ASENSIO, R., (2014). (7). 
que sea, de la LRBRL, sino frente a su derogación implícita. Añade, por último, que no tendría fundamento el agravio de limitar las competencias municipales, cuando los representantes municipales se hallan legitimados por sufragio directo, en comparación a las diputaciones provinciales, con legitimidad indirecta, para las que la Ley no contempla limitación alguna para el Legislador Sectorial en la atribución de competencias.

Este autor dedica atención singular al concepto de competencia impropia tomando en consideración si la calificación deben recibirla las nuevas competencias distintas de las propias o delegadas que un municipio pretenda ejercer una vez promulgada la ley o, si por el contrario, la promulgación obliga a revisar las impropias ejercidas hasta entonces y solicitar los informes vinculantes de inexistencia de duplicidad y garantía de sostenibilidad que permitan su ejercicio. En sentido análogo a la interpretación formulada para ver si el listado del artículo 25.2 permitía atribuir competencias propias a los municipios por parte de las comunidades autónomas, al FEMP, también en este caso, dada su similitud, considera sobrevenida la impropiedad de determinadas competencias, por ejemplo, las ejercidas al acaparo de la cláusula general del artículo 25.1 o las complementarias del artículo 28. Así, el profesor Jiménez Asensio recuerda que, a diferencia del reconocimiento implícito de competencias propias municipales en materias no recogidas en el artículo 25.2, el Ministerio de Hacienda y Administraciones Públicas sí considera la necesidad de solicitar informes para las competencias distintas de las propias o delegadas. El problema que se plantea es la aplicación retroactiva del artículo 7.4. Esta posibilidad, a juicio del referido autor, encuentra una dificultad derivada de la alusión al ejercicio de nuevas competencias, lo que supondría que las hasta ese momento ejercidas quedarían liberadas de la obligación de solicitud de informes autonómicos para su ejercicio; como en el caso del listado previsto en el artículo 25.2, la promulgación de una ley que modifica la anterior no permite desconocer los títulos competenciales que legitimaban el ejercicio de estas competencias pues, según el sistema de fuentes del Derecho, las leyes, salvo que haya excepción expresa y conforme al artículo 2.3 del Título Preliminar del Código Civil ("Las leyes no tendrán efecto retroactivo, si no dispusieren lo contrario"), se aplican a partir de la entrada en vigor de la Ley, es decir se aplican "pro futuro". Como nada dice la Ley sobre su aplicación pretérita ha de concluirse afirmando su carácter irretroactivo. Manifestación de esta aplicación pro futuro es la necesidad de suprimir las competencias impropias al elaborar un plan económico-financiero.

Pese a estos fundamentos jurídicos, el profesor Jiménez Asensio recomienda la promulgación de normativa autonómica que aclare el significado de competencia impropia y la necesidad de solicitud de expediente, tarea que ya han iniciado (a la fecha de cierre de estas líneas) cuatro comunidades autónomas en España, como veremos a continuación.

\section{NORMATIVA AUTONÓMICA DE BLINDAJE DE COMPETENCIAS MUNICIPALES FRENTE A LA LRSAL.}

Tal y como recomienda el profesor Jiménez Asensio, y ante la gran incertidumbre provocada por la LRSAL, a la fecha de cierre de estas líneas, cuatro comunidades autónomas han aprobado diferentes normas (Ley, Decreto-Ley, incluso Circular) con el fin de precisar y aclarar algunos de los temas para los que la Ley no ofrece una interpretación clara o una aplicación inequívoca.

El denominador común de esta normativa autonómica es tratar de desactivar el propósito de la LRSAL: sólo serán competencias municipales impropias las que, una vez promulgada la ley, no cuenten con habilitación del Legislador Sectorial correspondiente; en lo relativo a Servicios Sociales, Sanidad y Educación la calificación de esas competencias queda condicionada a la modificación de la financiación autonómica.

Como advirtiera en su día el profesor Muñoz Machado:

El legislador estatal no tiene el dominio de las competencias ni autonómicas ni locales. Por tanto, no puede imponer una regla que está inspirada en la idea de evitar entrecruzamientos de competencias... en el marco constitucional en el que nos movemos y considerando la jurisprudencia constitucional referida, el carácter exclusivo o no de las competencias provinciales, municipales o de las comunidades autónomas no depende de decisiones del legislador estatal22.

En efecto, el pretendido (por el Legislador Estatal) efecto armonizador de la LRSAL y su intención de conseguir la finalidad de "una Administración, una competencia", objetivos ambos más que loables en un principio, se le han vuelto en contra al Legislador Estatal, ya que el efecto provocado por la entrada en vigor de la LRSAL en algunas comunidades autónomas ha sido justamente el contrario, al haberse aprobado 4 normas de diverso rango, que son las siguientes:

- CIRCULAR de 11 de marzo de 2014, de la Directora de Relaciones con las Administraciones Locales y Registros Administrativos del País Vasco, referente al sistema de ordenación de las competencias municipales y al

22 Vid., MUÑOZ MACHADO, S., (2014). (10).

REALA, n 2 julio-diciembre 2014, ISSN: 1989-8975 - DOI: http://dx.doi.org/10.24965/reala.v0i2.10192 
régimen foral vasco, tras la entrada en vigor de la Ley 27/2013, de 27 de diciembre, de Racionalización y Sostenibilidad de la Administración Local.

- DECRETO-LEY 1/2014, de 27 de marzo, de medidas urgentes para la garantía y continuidad de los servicios públicos en Castilla y León, derivado de la entrada en vigor de la Ley 27/2013, de 27 de diciembre, de racionalización y sostenibilidad de la Administración Local.

- $\quad$ DECRETO-LEY 7/2014, de 20 de mayo, de la Junta de Andalucía, por el que se establecen medidas urgentes para la aplicación de la Ley 27/2013, de 27 de diciembre, de racionalización y sostenibilidad de la Administración Local.

- LEY Gallega de medidas urgentes derivadas de la entrada en vigor de la Ley 27/2013, de 27 de diciembre, de Racionalización y Sostenibilidad de la Administración Local, aprobada por el Parlamento de Galicia el 27 de mayo de 2014 .

De esta forma, se constata que la LRSAL, además de provocar un efecto completamente contrario al inicialmente previsto de unificación de criterios en todos los territorios, ha dado lugar a un hecho insólito hasta la fecha en nuestro ordenamiento jurídico, como es el que el Legislador Sectorial (en este caso, autonómico, y de diverso signo político), para la correcta aplicación de la Normativa Básica (la LRSAL) esté aprobando normativa, no de desarrollo (que sería lo normal y habitual), sino de "aclaración" o "interpretación” de algunas cuestiones polémicas o no suficientemente claras en la Ley Básica. Y además, y como es lógico, lo está haciendo "con carácter urgente", dado que se trata de cuestiones que no pueden esperar a la tramitación ordinaria de la referida normativa.

A este respecto, conviene tener muy presente lo que ha señalado nuestro Tribunal Constitucional en diversas ocasiones, en relación con los conceptos de legislación básica del Estado y de legislación autonómica complementaria o de desarrollo.

Afirma nuestro Tribunal Constitucional (entre otras, en la STC 102/1995, de 26 junio, RTC 1995/102), lo siguiente:

Lo básico consiste en el común denominador normativo para todos en un sector determinado... Lo básico, como propio de la competencia estatal, cumple una función de ordenación mediante mínimos que han de respetarse en todo caso, pero que pueden permitir que las Comunidades Autónomas con competencias en la materia establezcan niveles de protección más altos, como se dijo en la STC 170/1989.

El recíproco engranaje de la competencia estatal y de las autonómicas en la materia, visto así, lleva a la convicción de que lo básico tiene aquí simultáneamente carácter mínimo, como patrón indispensable para la protección del medio ambiente, fuera de cuyo núcleo entran en juego las normas que lo complementan y lo desarrollan, con la ejecución, sin fisura alguna de ese entero grupo normativo. Se trata pues, de una estratificación de la materia por niveles, donde el estatal ha de ser suficiente y homogéneo, pero mejorable para adaptarlo a las circunstancias de cada Comunidad Autónoma.

Por tanto, en el caso que nos ocupa podríamos hablar de una situación más que "anómala", donde entiende el Legislador Sectorial (Autonómico), que la normativa básica aprobada no es suficiente ni homogénea, por lo que en lugar de aprobar su normativa para mejorar y adaptar la Ley Básica a sus propias circunstancias, está aprobando una normativa para aclarar algunos de los términos de la misma y, en último término, para blindarse en su territorio de la aplicación de los mismos.

Este hecho, insólito hasta la fecha en nuestro ordenamiento jurídico, está dando lugar a otro también bastante curioso, como es el diferente rango normativo escogido para cumplir con esa finalidad por las cuatro comunidades autónomas: una Circular en el País Vasco, un Decreto-Ley en Castilla y León y Andalucía, y una Ley en Galicia.

En todo caso, y a nuestro entender, se puede afirmar que esta normativa autonómica aprobada hasta la fecha reviste un carácter más reglamentario que legislativo pese a que la lectura autonómica suponga un cambio radical en las aspiraciones del legislador estatal. Esto, claro está, es toda una paradoja también difícil de explicar y comprender.

De las cuatro normas autonómicas, la que nos parece más completa y desarrollada es la andaluza, esto es, el Decreto-Ley 7/2014, de 20 de mayo, de la Junta de Andalucía, por el que se establecen medidas urgentes para la aplicación de la Ley 27/2013, de 27 de diciembre, de racionalización y sostenibilidad de la Administración Local, de la que nos parecen dignos de mención los siguientes aspectos. 
En primer lugar, hay que partir de la base de que la intención última del Gobierno de la Junta de Andalucía con la aprobación de esta normativa es, en palabras de su Vicepresidente (y a la sazón, Consejero de Administración Local), "blindar las competencias de los ayuntamientos andaluces frente a la reforma de la Administración local" operada por la LRSAL, "de tal modo que se salvaguarde el amplio autogobierno que la legislación autonómica garantiza a los municipios, frente a la recentralización planteada por el Estado”.

Más en concreto, el objetivo perseguido por el ejecutivo andaluz es el de blindar las competencias municipales previstas en las conocidas como "leyes locales andaluzas". Y es que, como ya tuvimos ocasión de poner de manifiesto en su momento ${ }^{23}$, con la aprobación de las conocidas como "Leyes Locales en Andalucía” (la Ley 5/2010, de 11 de junio, de Autonomía Local para Andalucía²4 (en adelante, la "LAULA”) y la Ley 6/2010, de 11 de junio, reguladora de la Participación de las Entidades Locales en los tributos de la Comunidad Autónoma de Andalucía 25 (en adelante, la “PICA”), Andalucía se colocó ya en el año 2010 a la vanguardia de España en desarrollo del nuevo régimen local, al no existir hasta la fecha ninguna otra comunidad autónoma que hubiese abordado con tanta decisión y de forma tan ambiciosa la definición de un nuevo marco legal para sus entidades locales, mejorando la hacienda de los mismos a través de un fondo de nivelación incondicionado, que les ha permitido además priorizar sus políticas.

Como bases jurídicas, el Decreto-Ley andaluz parte de la previsión del artículo 60 del Estatuto de Autonomía de Andalucía, que otorga a la comunidad competencias exclusivas sobre régimen local; la doctrina consolidada del TC, que encomienda a la legislación básica estatal la garantía de la autonomía local, y la propia Disposición Adicional Tercera de la LRSAL, que hace referencia expresa al respeto a las atribuciones exclusivas de las comunidades autónomas. Este último precepto ha marcado también la interpretación de la aplicación de la reforma local en las otras normas aprobadas recientemente en Castilla y León, Galicia y el País Vasco.

En cuanto a la consideración de las competencias propias, el Decreto-Ley andaluz establece que siguen siendo competencias propias de los ayuntamientos las 29 que recoge el Estatuto andaluz y desarrolla la LAULA.

Por otra parte, interpreta la aplicación de la reforma local en el sentido de acotar al máximo el concepto de duplicidad para no interferir en la capacidad de autogobierno. De este modo, se considera que sólo se dará esta circunstancia si las competencias que los municipios ejercen coinciden con las de la Junta de Andalucía en el mismo territorio y para la misma población, y si no son complementarias. El informe sobre inexistencia de duplicidades o de ejecución simultánea del mismo servicio público con otra administración, lo emitirá la consejería competente en la materia de que se trate. Si es favorable, se remitirá a la Consejería de Hacienda y Administración Pública, que a su vez elaborará el dictamen sobre sostenibilidad financiera. Por tanto, se establece una suerte de control de oportunidad para evaluar la ausencia de duplicidad a la hora de valorar si se trata de una nueva competencia o de la revisión de otra ejercida con anterioridad a la aprobación de la Ley. El artículo 3 enumera los criterios que la consejería competente valorará para constatar la existencia o no de ejecución simultánea del mismo servicio; basta reparar en el contenido de los tres apartados, las características del servicio o la actividad pública de que se trata, el alcance de las prestaciones que se generarán a favor de la ciudadanía y las condiciones para las políticas de fomento, para constatar la amplia discrecionalidad de la comunidad autónoma a la hora de apreciar la concurrencia de duplicidad.

Además de lo anterior, el Decreto-Ley andaluz aclara las incertidumbres jurídicas que plantea la aplicación de la LRSAL y evita los vacíos competenciales que pueden producirse hasta el pronunciamiento del TC sobre el recurso de la Junta de Andalucía, especialmente en servicios sociales, sanidad y educación. En esta línea, se regula el procedimiento para el ejercicio de las competencias denominadas “impropias” o por delegación, así como el relativo a la adaptación de los convenios firmados entre las administraciones autonómica y local para la financiación de las mismas.

Los informes preceptivos en ambos procedimientos no serán necesarios si las competencias se vienen ejerciendo desde antes de la entrada en vigor de la reforma local aprobada por el Gobierno central, salvo que se detecte la ejecución simultánea de un mismo servicio público o el ayuntamiento no cuente con financiación.

En relación con ello, cabría resaltar como cuestiones más relevantes, en primer lugar, el destacado papel atribuido a la intervención municipal, que va en contra de la recomendación del Consejo de Estado en su Dictamen sobre el Anteproyecto de LRSAL. Es cierto que la decisión final corresponde a la comunidad autónoma, pero no cabe subestimar los tres informes requeridos al interventor ${ }^{26}$.

\footnotetext{
23 Vid., NAVARRO RODRÍGUEZ, P., y MARTÍNEZ VIDAL, F., (2010). (12).

24 La Ley $13083 / 2010$.

25 La Ley 13173/2010.

26 Sobre este tema, vid., in extenso, FUEYO BROS, M., (2014). (5).
} 
Por último, y como nota también a destacar, estaría la conveniencia de, a la vista de la definición legal del concepto de duplicidad (ejecución simultánea), la trascendencia jurídica sobre la relación de la nueva competencia con una anterior a la promulgación de la ley o como dice el Decreto-Ley andaluz sea meramente una ampliación o complemento de una ya ejercida. Motivos para calificar de control de oportunidad un informe tan poco propicio a su remisión a criterios de legalidad. En este sentido, debemos destacar que el profesor Velasco ha propuesto un criterio para distinguir entre la validez y aplicación de la LRSAL y la legislación autonómica, que ha sido el que ha guiado el decreto andaluz.

En todo caso, hay que tener en cuenta que a la fecha de cierre de estas líneas, el Ministro de Hacienda y Administraciones Públicas acaba de anunciar la convocatoria urgente de la Comisión mixta Gobierno-Junta de Andalucía, por lo que probablemente en la misma se aclaren algunas de las muchas dudas e incertidumbres que aún existen en cuanto a la aplicación de la LRSAL en Andalucía.

\section{BIBLIOGRAFÍA}

1. CARRILLO DONAIRE, J.A., Y NAVARRO RODRÍGUEZ, P., (COORDS.) (2014): La Reforma del Régimen Jurídico de la Administración Local. El nuevo marco regulatorio a la luz de la Ley de racionalización y sostenibilidad de la Administración Local, La Ley-El Consultor de los Ayuntamientos, Madrid.

2. CARRO FERNÁNDEZ-VALMAYOR, J.L., (1999/2000): La cláusula general de competencia municipal. Anuario de Gobierno Local.

3. DÍAZ LEMA, J.M., (2012): El Anteproyecto de Ley de racionalización y sostenibilidad de la Administración local: ¿cambio de rumbo en la Administración local española? Anuario de Gobierno Local.

4. FEDERACIÓN ESPAÑOLA DE MUNICIPIOS Y PROVINCIAS (2013): Alegaciones de la FEMP al Anteproyecto de Ley de Racionalización y Sostenibilidad de la Administración Local.

5. FUEYO BROS, M., (2014): «Fortalecimiento de la figura del interventor. Función interventora y control interno: el control evanescido", en La Reforma del Régimen Jurídico de la Administración Local. El nuevo marco regulatorio a la luz de la Ley de racionalización y sostenibilidad de la Administración Local, J.A Carrillo Donaire y P. Navarro Rodríguez (Coords.), La Ley-El Consultor de los Ayuntamientos, Madrid.

6. GARCIA RUBIO, F., (2014): «La provincia como entidad local. Un análisis tras la Ley de Racionalización», La reforma de 2013 del régimen local español, J.A. Santamaría Pastor (Dir.), Fundación Democracia y Gobierno Local, pp. 195-303.

7. JIMÉNEZ ASENSIO, R., (2014): Vademécum sobre la Ley de Racionalización y Sostenibilidad de la Administración Local: 100 cuestiones sobre su aplicación. Federació de Municipis de Catalunya, Barcelona.

8. MEDINA GUERRERO, M., (2014): La Reforma del Régimen Local, Tirant Lo Blanch, Barcelona.

9. MUÑOZ MACHADO, S. (Dir.), (2011): Tratado de Derecho Municipal I, $3^{\mathrm{a}}$ ed., lustel, Madrid.

-, (2013): «Ocho mil millones de euros de ahorro: la compleja reforma de la Administración Local; Círculo Cívico de Opinión, 22 de abril de 2013».

10. NAVARRO RODRÍGUEZ, P., (2007): «Las 50 primeras preguntas del Concejal», Estudio de Divulgación núm. 130, Centro de Estudios Municipales y de Cooperación Internacional (CEMCI), Granada.

-, Con MARTíNEZ VIDAL, F., (2010): Las 111 preguntas más frecuentes de los Concejales en Andalucía, Centro de Estudios Municipales y de Cooperación Internacional (CEMCI), Granada.

11. VELASCO CABALLERO, F., (2014): «Títulos competenciales y garantía constitucional de autonomía local en la Ley de Racionalización y Sostenibilidad de la Administración Local», La reforma de 2013 del régimen local español, J.A. Santamaría Pastor (Dir.), Fundación Democracia y Gobierno Local, pp. 75 a 136.

12. ZAFRA VÍCTOR, M., (2008): «Autonomía local: un pilar en la organización del Estado de las Autonomías», en ZAFRA VÍCTOR, M. (Coord.), Relaciones institucionales entre Comunidades Autónomas y Gobiernos Locales, IAAP, Sevilla.

-, (2013a): «Análisis y valoración del Anteproyecto de Ley para la racionalización y sostenibilidad de la Administración local», en SALVAdor CRESPO, M., ZAFRA Víctor, M. y MEdina GUerRero, M., Reforma de la Ley de Régimen Local, Fundación Centro de Estudios Andaluces, Sevilla.

—, (2013b): «Esta reforma local empobrece la democracia», Diario El País, 1 de agosto de 2013. 
—, (2014): «Análisis y valoración sobre la constitucionalidad de la Ley de racionalización y sostenibilidad de la Administración local», en La Reforma del Régimen Jurídico de la Administración Local. El nuevo marco regulatorio a la luz de la Ley de racionalización y sostenibilidad de la Administración Local, J.A Carrillo Donaire y P. Navarro Rodríguez (Coords.), La Ley-El Consultor de los Ayuntamientos, Madrid. 